\title{
Multi-Sensor Classification of Tennis Strokes
}

\author{
Damien Connaghan, Phillip Kelly and Noel E. O'Connor Mark Gaffney, Michael Walsh and Cian O'Mathuna \\ CLARITY: Centre for Sensor \\ Web Technologies \\ Dublin City University \\ Tyndall National Institute \\ Dyke Parade, Cork, Ireland \\ Email: mark.gaffney@tyndall.ie
}

Dublin 9, Ireland

Email: connagha@eeng.dcu.ie

\begin{abstract}
In this work, we investigate tennis stroke recognition using a single inertial measuring unit attached to a player's forearm during a competitive match. This paper evaluates the best approach for stroke detection using either accelerometers, gyroscopes or magnetometers, which are embedded into the inertial measuring unit. This work concludes what is the optimal training data set for stroke classification and proves that classifiers can perform well when tested on players who were not used to train the classifier. This work provides a significant step forward for our overall goal, which is to develop next generation sports coaching tools using both inertial and visual sensors in an instrumented indoor sporting environment.
\end{abstract}

\section{INTRODUCTION}

In this paper, we describe our approach to automatically index a tennis match based on strokes played using a single Wireless Inertial Measuring Unit (WIMU), which is attached to a tennis player's forearm (as shown in Figure 3). We classify the main types of tennis strokes (forehand, backhand and serves) played in a competitive match. Each WIMU contains accelerometer, gyroscope and magnetometer sensors, aligned with three principle axes. In our previous work on tennis stroke recognition, we classified the accelerometer data from the WIMUs only and the data set used was produced in a supervised environment, where several professional players performed a series of strokes from a fixed position [10]. This paper delivers a new contribution, which can classify tennis strokes performed in a competitive match, by players of different levels using either accelerometers, magnetometers or gyroscopes. The two-level classification process used in this paper can filter any player movements where they are not performing a tennis stroke whilst the second step classifies candidate tennis strokes into serves, backhands or forehands. We evaluate the accuracy of using accelerometer, gyroscope and magnetometer sensors to perform tennis stroke classification on previously unseen players.

Automatic detection of tennis events is necessary to reduce the time a coach will spend manually indexing a recorded match and the work in this paper is part of a wider system, which we have developed to automatically segment a tennis match into key events using both inertial and visual sensors in an instrumented environment. In previous publications, we outlined our work for detecting key tennis events in a match using visual sensors [5] [8] [10] and also on the development of a suitable sports coaching system [6] [7], where a coach can quickly retrieve key events from a tennis match. However this paper describes how inertial sensing can efficiently index tennis strokes. The advantage of using inertial sensing to index tennis strokes is of course that, visual sensing can suffer from self occlusion and player orientation issues, which are inherent in wide area scene analysis.

\section{RELATED WORK}

Lightweight inertial sensors are cheap and ubiquitous. Research into such sensors has focused on recognition of human actions. For example, microphones are combined with threeaxis accelerometers by Ward et al. to determine the activities of a person in maintenance and assembly tasks [13].

There has been plenty previous work on tennis stroke recognition using visual sensing [5] [8] [4], but the research field of stroke classification using inertial sensors has not been as active. However, one such contribution from [3], investigates the possibility of using wearable gyroscope sensors for skill assessment and skill acquisition in a tennis serve. A markerbased method using the vicon motion capture system [2] was used to calculate the angular velocity of the upper arm internal rotation, wrist flexion, and shoulder rotation for a range of athletes during the first serve in tennis. Participants from amateur to elite participated in this study and results showed that the peak values of the upper arm internal rotation, wrist flexion, and shoulder rotation just before impact are indicative in classifying the participants' skill level. The correct positioning of three gyroscope sensors on the player's arm, to detect the same trends as those from the marker-based methods were then determined. As a result [3] envisaged that gyroscope sensors could be used for skill assessment and skill acquisition for a first tennis serve.

In our previous work to detect tennis strokes in an instrumented environment [10], we used visual and inertial sensors to recognise tennis strokes played from a fixed position on a tennis court. The cameras were positioned behind the player and action recognition was achieved using pie features of the players silhouette. Accelerometer magnitudes were also used to detect the temporal locations where the ball and racket collide and a $\mathrm{W}$-second window was extracted from the accelerometer data and classified into strokes played using either Support Vector Machine(SVM) classifiers or K-means nearest neighbor clustering. 


\section{INFRASTRUCTURE}

In collaboration with Tennis Ireland [1], the national governing body for Tennis in Ireland, we have instrumented an indoor tennis-court with a data-gathering infrastructure for use as a test-bed for sports and health research. This infrastructure includes overhead and baseline IP cameras with pan, tilt and zoom capability from which a tennis match can be digitally captured. In addition, the capture framework uses a custom, Tyndall developed TennisSense WIMU system which is based on Tyndall's $25 \mathrm{~mm}$ Mote platform [11]. This provides a small, wearable and low cost method for instrumenting human subjects to provide high speed motion data [10]. This system consists of up to 6 Wearable WIMUs connecting wirelessly to a base station using a $2.4 \mathrm{GHz}$ RF system, which in turn connects to the PC over USB, appearing as a serial device and delivering data as a simple text stream [9]. In this work we focus on using a single WIMU placed onto the player's forearm during a competitive match as shown in Figure 3.

\section{SYSTEM OVERVIEW}

In this section, we give an overview of our tennis stroke detection system using inertial measuring units only. As each WIMU contains accelerometers, gyroscopes and magnetometers, we can detect strokes played using any of the three sensors.

\section{A. Tennis Stroke Detection}

A WIMU sensor placed on a players dominant arm will register a spike in its accelerometer data due to the impact of the ball on the tennis racket. Detecting such data-spikes provides the temporal location of tennis strokes, as shown in Figure 1. To detect ball contact impacts, we first compute the acceleration magnitude for each sensor sample, simply by taking the length of the 3D acceleration vector. We select the value with the largest absolute magnitude in the data. A $\mathrm{W}$-second window around this peak is extracted to represent a candidate stroke in progress. Adopting a greedy approach, this window is removed from the data and the procedure is then repeated to find the remaining candidate strokes, until we have extracted all candidate strokes which generate an accelerometer magnitude of $3 \mathrm{~g}$ or above. Even the slightest of ball contact will always generate a magnitude of at least $3 \mathrm{~g}$ in force, so this is a suitable threshold for detecting candidate strokes.

However, there are other actions which a tennis player will perform during a match, that will generate a spike in accelerometer magnitude and therefore it is necessary to identify which of these spikes are candidate tennis strokes and which spikes are generated from a player performing a non tennis stroke. A non-stroke action can include using the racket to lift a ball of the court surface or twirling the racket in a players hands whilst waiting on an opponent to serve, which is in fact common. Also problematic are activities such as running, practice swings and arm movements performed during rest periods. Its for this reason that we use a two level classification system to classify candidate strokes.

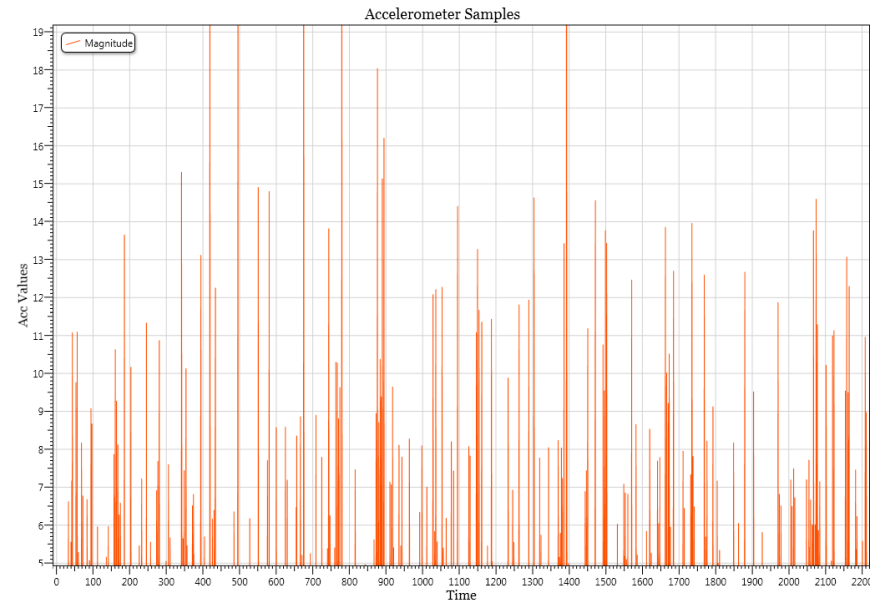

Fig. 1. Tennis stroke detection using accelerometer magnitude on dominant arm: red lines show detected strokes.

\section{B. Stroke Classification}

The classification of tennis strokes is accomplished in two steps as shown in Figure 2. The top level of this process filters non-stroke events, which generate an accelerometer magnitude from various arm movements during a match. The second stage of the classification uses either accelerometers, magnetometers or gyroscopes to classify all the candidate strokes into either serves, backhands or forehands.

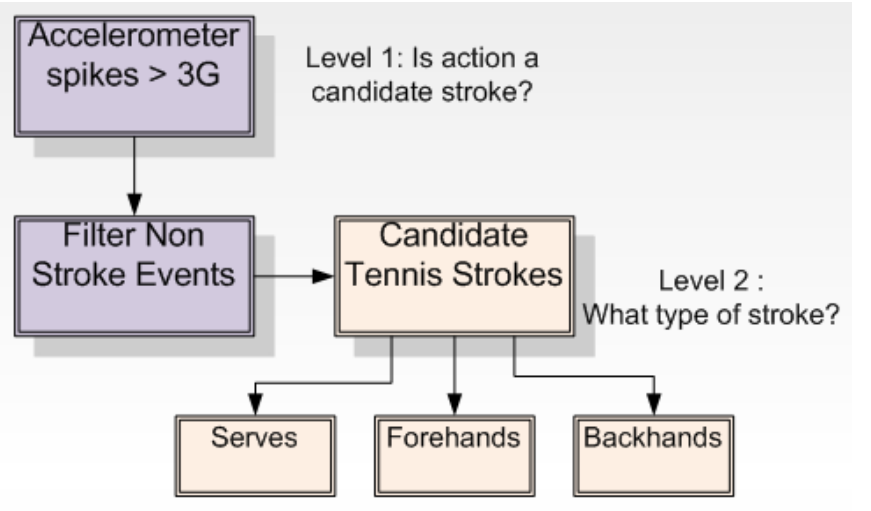

Fig. 2. Two-level classification: Step one filters noisy data and step two classifies the remaining candidate strokes. Both steps use naive Bayesian classifiers

1) Filtering Non-Tennis Stroke Events: To filter non-stroke events, we use the accelerometer data from 8 players during a tennis match to create two global feature vectors, one global feature vector contained a mixture of serves, backhands and forehands as played in a competitive match. The second global feature vector contained all non-stroke events. The model was trained using a naive Bayesian classifiers, which are known to be effective at classifying instances with a high attribute list and have been successfully employed as binary classifiers in the past to filter email spam [12].

Each W-second accelerometer instance that had a magnitude of $3 \mathrm{~g}$ or greater was feed into the binary classifier and any instance which was predicted as a non-stroke event was filtered 
from the dataset. The remaining candidate strokes were passed to level two of the classification process to predict if the stroke is a backhand, forehand or serve.

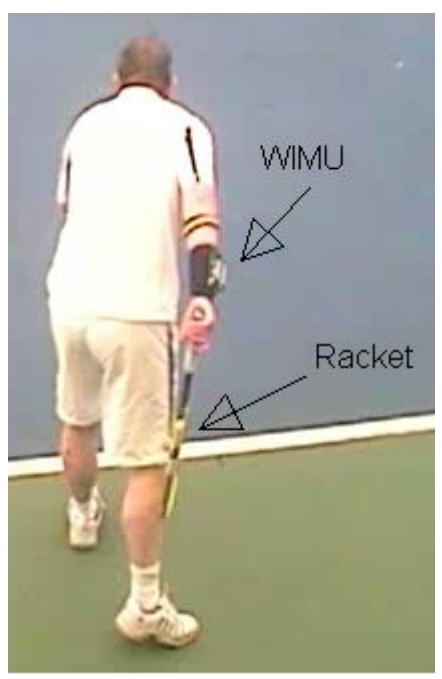

Fig. 3. WIMU as positioned on a player

2) Candidate Stroke Classification: To classify candidate strokes into serves, backhands and forehands, we first trained three classifiers for each stroke. For each serve, we trained a classifier with accelerometer data from a subset of serves by various players, then a second serve classifier was trained with gyroscope data of serves and a third serve classifier was trained with the magnetometer data for serves. Similarly, three classifiers were built for forehands and backhands.

Having filtered out all noise from the data we have the temporal locations of all candidate strokes that a single player has performed during a match. Using the temporal locations we can select each candidate stroke in turn. For each candidate stroke, we then select the accelerometer data at this time and compare it to the serve classifier, forehand classifier and backhand classifier for the accelerometer data. The model is then able to predict if the candidate stroke belongs to the serve class, forehand class or backhand class. An identical approach is used for employ the gyroscopes and magnetometer classifiers.

\section{EXPERIMENTS}

In this section we discuss the main experiments and also the results and findings from these experiments

\section{A. Filtering Non-Stroke Events}

Step one in the two stage classification process detects which spikes in the accelerometer data are likely to be tennis strokes and which can be considered non-strokes. To verify the accuracy of this binary classifier, we performed 10 fold cross validation on the entire dataset of accelerometer instances from all players which were above $3 \mathrm{~g}$ in magnitude.

As the results prove, this filtering process has a very high accuracy rate at removing any non-stroke events. This filtering is necessary to create accurate candidate stroke classification in step 2 of this classification process. With respect to Table I, precision is the number of correct results returned, divided by the number of all returned results. Recall is the number of correctly classified strokes divided by the number of results that should have been returned, while Acc. is the percentage measure of the correctly classified instances.

\begin{tabular}{|c|c|c|c|c|}
\hline Category & $\#$ & Precision & Recall & \% Acc. \\
\hline Candidate Strokes & 2090 & 0.688 & 0.89 & 85 \\
\hline Non-Candidate Stroke events & 5749 & 0.95 & 0.83 & 85 \\
\hline
\end{tabular}

TABLE I

DETECTING ALL NON TENNIS STROKE EVENTS WHICH ARE GENERATING A SPIKE IN THE MAGNITUDE OF ACCELEROMETER DATA.

\section{B. Stroke Recognition}

A series of experiments on stroke recognition are reported in Table II, which shows results for advanced (Adv.), intermediate (Inter.), and novice players. The acceleromoter stroke classification section in Table II illustrates how the stroke classifiers performed when trained on 7 players and tested on a unseen player. It also illustrates the results when trained on a random 4 players were then tested on an unseen player. The gyroscope section and magnetometer section in Table II report results in a similar way.

1) Testing classifiers on players not in the training set : To evaluate how accurate each sensor is at predicting strokes from a player who is not in the training set, we trained the classifiers with 7 players and tested on the remaining unseen player. For each player the classifiers were retrained using all the other players to find if stroke classification can be achieved by testing on an unseen player. The results are displayed in the 7 player training size section in Table II.

As the results in Table II prove stroke classification can indeed be accurately achieved without training the classifiers on the player who performed the candidate stroke. It should also be noted that since the classifiers were trained on a variety of players from different skill levels that to achieve $79 \%$ accuracy, as was the case with the accelerometer classifiers is very encouraging. As expected however the gyroscopes perform the worst of the 3 sensors, this is because the measure of temporal orientation during a given stroke will be significantly different between players of different skill levels. This is in contrast to a tri-axis accelerometer, which measures acceleration on three planes, which will be more effective at classifying strokes from different skill levels.

2) Evaluating classification performance as training size decreases: We trained the stroke classifiers on 4 players and tested on an unseen player. The results from this experiment were compared to the results from training on 7 players. With accelerometers and gyroscopes, there was no significant decrease between training on 7 players or training on 4 players. However, the magnetometers display a $10 \%$ decrease in performance as the training set is reduced to 4 players. 


\begin{tabular}{|c|c|c|c|}
\hline \multicolumn{2}{|l|}{ Training Size: } & $\begin{array}{l}7 \text { players } \\
2543 \text { strokes }\end{array}$ & $\begin{array}{l}4 \text { Players } \\
\text { strokes }\end{array}$ \\
\hline Player & Test Strokes & $\%$ & $\%$ \\
\hline \multicolumn{4}{|c|}{ Accelerometer Stroke Classification } \\
\hline Adv. Player A & 597 & 77 & 68 \\
\hline Adv. Player B & 197 & 80 & 80 \\
\hline Adv. Player C & 177 & 82 & 82 \\
\hline Inter. player D & 220 & 62 & 62 \\
\hline Inter. player E & 333 & 80 & 77 \\
\hline Inter. player $\mathrm{F}$ & 325 & 78 & 82 \\
\hline Novice player $\mathrm{G}$ & 163 & 78 & 88 \\
\hline Novice player $\mathrm{H}$ & 225 & 84 & 80 \\
\hline Overall Accuracy & & $79 \%$ & $80 \%$ \\
\hline \multicolumn{4}{|c|}{ Gyroscope Stroke Classification) } \\
\hline Adv. Player A & 597 & 48 & 64 \\
\hline Adv. Player B & 197 & 84 & 85 \\
\hline Adv. Player C & 177 & 82 & 83 \\
\hline Inter. player D & 220 & 46 & 50 \\
\hline Inter. player $\mathrm{E}$ & 333 & 73 & 59 \\
\hline Inter. player $\mathrm{F}$ & 325 & 71 & 70 \\
\hline Novice player $\mathrm{G}$ & 163 & 71 & 72 \\
\hline Novice player $\mathrm{H}$ & 225 & 78 & 73 \\
\hline Overall Accuracy & & $76 \%$ & $71 \%$ \\
\hline \multicolumn{4}{|c|}{ Magnetometer Stroke Classification) } \\
\hline Adv. Player A & 597 & 72 & 70 \\
\hline Adv. Player B & 197 & 78 & 74 \\
\hline Adv. Player C & 177 & 78 & 79 \\
\hline Inter. player D & 220 & 48 & 50 \\
\hline Inter. player $\mathrm{E}$ & 333 & 87 & 86 \\
\hline Inter. player $\mathrm{F}$ & 325 & 74 & 76 \\
\hline Novice player $\mathrm{G}$ & 163 & 78 & 75 \\
\hline Novice player $\mathrm{H}$ & 225 & 81 & 75 \\
\hline \multicolumn{2}{|l|}{ Overall Accuracy } & $76 \%$ & $75 \%$ \\
\hline
\end{tabular}

TABLE II

TENNIS STROKE CLASSIFICATION, TESTING ON A PLAYER NOT IN TRAINING SET. ONE CLASSIFIER IS TRAINED ON 7 PLAYERS AND THE OTHER IS TRAINED ON A RANDOM 4 PLAYERS TO ILLUSTRATE PERFORMANCE DECREASE AS THE TRAINING SET DECREASES.

\begin{tabular}{|c|c|}
\hline Sensor Fusion & Overall Accuracy \% \\
\hline Accelerometer \& Gyroscope & 82.5 \\
\hline Accelerometer \& Magnetometer & 86 \\
\hline Gyroscope \& Magnetometer & 88 \\
\hline Accelerometer \& Gyroscope \& Magnetometer & 90 \\
\hline
\end{tabular}

TABLE III

TO ANALYSE THE BENEFITS OF SENSOR FUSION BEFORE CLASSIFICATION, WE PERFORMED EXPERIMENTS TRAINING FROM 7 PLAYERS AND TESTING ON AN UNSEEN PLAYER.

3) Sensor Fusion Comparison: In this experiment we trained the classifiers with a combination of the data from three sensors to identify if stroke recognition performance is effected using sensor fusion. The classifiers were trained using seven players and again tested on a player not in the training set. Using this leave one player out approach, we retrained the classifiers for each player. The results can be seen in Table III. Interestingly, we discovered that using a combination of accelerometer, gyroscope and magnetometer sensors gives an overall stroke recognition performance of $90 \%$. This accuracy rate is $10 \%$ higher than that of accelerometer classification, which gave the highest results in the single sensor classifica- tion in Table II.

\section{CONCLUSION}

Overall, accelerometers perform the best of the three sensors at stroke recognition when trained on multiple players, but when we fuse the data from the three sensors and train the classifiers on a large data set this gives the best performance as illustrated in Table III.

In the future we plan to fuse this inertial stroke recognition system with our visual event recognition system [5] to improve the performance of detecting tennis events and ultimately create a tennis coaching system which will automatically segment a game into key events and allow the coaches quickly retrieve interesting locations in a game.

\section{ACKNOWLEDGMENT}

This work is supported by Science Foundation Ireland under grant 07/CE/I1147 and the National Access Program.

\section{REFERENCES}

[1] Tennis ireland. http://www.tennisireland.ie/.

[2] Vicon motion capture systems. http://www.vicon.com/.

[3] A. Ahmadi, D. Rowlands, and D. A. James. Towards a wearable device for skill assessment and skill acquisition of a tennis player during the first serve. page 29136, 2010.

[4] T. Bloom and P. Bradley. Player tracking and stroke recognition in tennis video. In Proceedings of the WDIC, pages 93-97, 2003.

[5] D. Conaghan, P. Kelly, and N. E. O'Connor. Game, shot and match: Event-based indexing of tennis. In 9th International Workshop on Content-Based Multimedia Indexing, pages 96-102, 2011.

[6] D. Conaghan and N. E. O'Connor. An intuitive user interface for visual sports coaching. In Proceedings of the Irish Human Computer Interaction (iHCI) Conference, pages 141-142, 2010.

[7] D. Connaghan, S. Hughes, G. May, K. O’Brien, P. Kelly, C. Ó Conaire, N. E. O'Connor, D. O'Gorman, G. Warrington, A. F. Smeaton, and N. Moyna. A sensing platform for physiological and contextual feedback to tennis athletes. In Body Sensor Networks (BSN) Workshop, pages 224-229, 2009.

[8] D. Connaghan, C. O'Conaire, P. Kelly, and N. E. O'Connor. Recognition of tennis strokes using key postures. In ISSC 2010 - 21st IET Irish Signals and Systems Conference, Cork, Ireland, 23-24 June 20102010. Institution of Engineering and Technology, Institution of Engineering and Technology.

[9] M. Gaffney, B. O'Flynn, A. Mathewson, J. Buckley, J. Barton, P. Angove, J. Vcelak, C. O'Connaire, G. Healy, K. Moran, N. E. O'Connor, S. Coyle, P. Kelly, B. Caulfield, and L. Conroy. Wearable wireless inertial measurement for sports applications. In IMAPS-CPMT Poland Conference, 2009.

[10] C. Ó Conaire, D. Connaghan, P. Kelly, N. E. O'Connor, M. Gaffney, and J. Buckley. Combining inertial and visual sensing for human action recognition in tennis. In Proceedings of the first ACM international workshop on Analysis and retrieval of tracked events and motion in imagery streams, pages 51-56, 2010.

[11] B. O'Flynn, S. Bellis, K. Delaney, J. Barton, S. C. O'Mathuna, A. M. Barroso, J. Benson, U. Roedig, and C. Sreenan. The development of a novel minaturized modular platform for wireless sensor networks. In Proceedings of the 4th international symposium on Information processing in sensor networks, IPSN '05, Piscataway, NJ, USA, 2005. IEEE Press.

[12] K.-M. Schneider. A comparison of event models for naive bayes anti-spam e-mail filtering. In Proceedings of the tenth conference on European chapter of the Association for Computational Linguistics Volume 1, EACL '03, pages 307-314, Stroudsburg, PA, USA, 2003. Association for Computational Linguistics.

[13] J. A. Ward, P. Lukowicz, G. Troster, and T. E. Starner. Activity recognition of assembly tasks using body-worn microphones and accelerometers. IEEE Trans. Pattern Anal. Mach. Intell., 28(10):15531567, 2006. 